\title{
Insuficiência Cardíaca em Grande Hospital Terciário de São Paulo
}

\author{
Antonio Carlos Pereira Barretto, Moacir Roberto Cuce Nobre, Maurício Wajngarten, Manoel \\ Femandes Canesin, Dalia Ballas, João Batista Serro-Azul
}

São Paulo, SP

\begin{abstract}
Objetivo - Verificar a incidência, principais causas, fatores desencadeantes ou de piora da insuficiência cardiaca (IC) no ano de 1995, no Instituto do Coração de São Paulo.

Métodos - Foram analisados os registros referentes a pacientes internados, obtidos do banco de dados da PRODESP. Analisaram-se nos pacientes com IC os dados: idade, sexo, diagnóstico principal e secundários, procedimentos executados ébitos. Para fim de análise, construíram-se tabelas de distribuição conforme o sexo, idade e diagnóstico principal. Análise de variância e teste do quiquadrado foram empregados para verificar diferença entre os grupos estudados.

Resultados -Dos pacientes internados (903 de 9620) 9,38\% apresentaram IC. As idades variaram de 2 dias a 98 (média 52,6) anos e a maioria era do sexo masculino $(60,4 \%)$. Miocardiopatia isquêmica (32,6\%), miocardiopatia dilatada $(25,8 \%)$ e valvopatias $(22,0 \%)$ foram as principais causas de IC. Foram submetidos à cirurgia, angioplastia, ou implante de marcapasso, 32,1\% dos pacientes, sendo os valvopatas na maioria submetidos à correção de sua cardiopatia de base $(63,3 \%)$. Houve maior incidência de múltiplos diagnósticos secundários com o aumento da idade. A mortalidade foi maior nos com idade $<20$ anos e nos $>80$ anos.

Conclusão - A incidência de IC foi de 9,38\%, sendo miocardiopatia isquêmica a causa mais freqüente. Foi possivel corrigir a causa da IC em 32,1\%. A mortalidade foi maior nas crianças provavelmente pela maior complexidade de sua cardiopatia e nos mais idosos devido à maior associação de diagnósticos secundários ou fatores agravantes.
\end{abstract}

Palavras-chave: insuficiência cardíaca, diabetes mellitus, fibrilação atrial

\section{Heart Failure in a Large Tertiary Hospital of São Paulo}

Purpose - To study the incidence, main causes, aggravating factors and secondary diagnoses of heart failure (HF) during 1995 at the Instituto do Coração of São Paulo.

Methods - Data from hospitalized patients according to the PRODESP data base were analyzed. The following data were studied: age, sex, principal and secondary diagnoses, surgical procedures and mortality. To analyze the data, tables according to sex, age and main cause were built. Analysis of variance and t test were employed to verify differences between groups.

Results - In 1995, 903 out of 9620 patients were hospitalized due to $H F$. The majority were male $(60.4 \%)$ and the patients' age was between two days and 98 years old (mean 52.6). Ischemic (32.6), dilated (25.8\%) and valvar heart disease (22\%) were the main causes of HF. $32.1 \%$ were submitted to correction of the HF main cause, specially those with valvar heart disease (62.3\%). There was greater incidence of multiple diagnoses in aged patients. The mortality was greater in patients younger than 20 and in those older than 80 years old.

Conclusion - The incidence of HF at INCOR during 1995 was $9.38 \%$. Ischemic myocardiopathy was the most frequent HF cause. The mortality was greater among children, probably because of heart disease complexity and, in the above-80 group due to the greater comorbidity.

Key-words: heart failure, diabetes, atrial fibrillation

Arq Bras Cardiol, volume 71 (n 1), 15-20, 1998

Instituto do Coração do Hospital das Clínicas - FMUSP

Correspondência: Antonio Carlos Pereira Barretto - Incor - Divisão de Cardiologia Social - Av. Dr. Enéas C. Aguiar, 44 - 05403-000 - São Paulo, SP

Recebido para publicação em 7/1/98

Aceito em 19/3/98
Insuficiência cardíaca (IC) tem uma prevalência de 1 a $2 \%$ na população geral ${ }^{1,2}$ e, entre nós, é a maior causa de internação dentre os diagnósticos cardiológicos no Sistema Único de Saúde (SUS) ${ }^{3}$. Apesar dos avanços diagnósticos e terapêuticos, sua incidência parece estar aumentando. 
Esta tendência se deve ao prolongamento da sobrevida com o manuseio da cardiopatia de base, oferecendo a oportunidade do surgimento da IC, fase final comum a todas as cardiopatias ${ }^{4}$. Dados dos Estados Unidos permitem supor que haja mais de quatro milhões e meio de pacientes com IC, ocasionando cerca de um milhão de internações por ano.

A IC é doença muito limitante, necessitando seus portadores, com certa frequiência, de internações para compensação, especialmente para os casos de maior comprometimento cardíaco ${ }^{5,6}$.

Apesar do reconhecimento crescente sobre a importância que a IC vem adquirindo nos últimos anos, no Brasil, há carência de dados epidemiológicos sobre a doença. Assim, procuramos analisar aspectos concernentes à IC dentre os pacientes internados em nossa Instituição, durante o período de um ano, como a freqüência, provável etiologia, associação com diagnósticos secundários ou agravantes, diferenças conforme o sexo e idade e mortalidade hospitalar.

\section{Métodos}

Foram analisados os dados de pacientes internados no INCOR, em 1995, obtidos do banco de dados da PRODESP, a partir de relatórios de internação, altas e óbitos, fornecidos pelo Serviço de Arquivo Médico e Estatística (SAME).

Dos casos com diagnóstico de IC, foram analisados: idade, sexo, diagnóstico principal, diagnósticos secundários, procedimentos executados (cirurgia, angioplastia ou implante de marca passo) e óbitos, procurando-se verificar a etiologia da IC, possíveis fatores desencadeantes ou agravantes.

Os pacientes foram agrupados por faixa etária em seis grupos: menores de 19, de 20 a 39, 40 a 59, 60 a 79 anos e maiores de 80 anos.

Os diagnósticos da cardiopatia principal, para fim de análise, foram reunidos em: cardiopatia congênita, hiperten- são arterial (HA), miocardiopatia dilatada, miocardiopatia isquêmica, miocardiopatia restritiva, valvopatia e outros. Como miocardiopatia dilatada foram incluídos os seguintes diagnósticos: doença de Chagas, miocardite, miocardiopatia alcoólica, taquimiocardiopatia e cardiomiopatia periparto. Pacientes com diagnóstico principal de infarto do miocárdio foram classificados como portadores de miocardiopatia isquêmica. Compuseram o grupo de miocardiopatia restritiva, pacientes com diagnóstico de endomiocardiofibrose, amiloidose e miocardiopatia restritiva idiopática e, como outras etiologias, os casos de hipertireoidismo, aneurisma dissecante de aorta, anemia, enfisema pulmonar, hipertensão pulmonar, fístula arterial, miocardiopatia hipertrófica, pericardite constritiva, bem como os casos em que não havia referência de cardiopatia bem definida que permitisse agrupálos com os já referidos diagnósticos principais.

São apresentadas as frequiências dos vários diagnósticos, os valores da média e desvio-padrão. Foram elaboradas tabelas com a distribuição de casos segundo o sexo, para diferentes faixas etárias e para diferentes diagnósticos da cardiopatia a fim de analisar possíveis correlações.

Realizaram-se análise de variância e teste de qui-quadrado para verificar se havia diferença entre os vários grupos estudados.

\section{Resultados}

No INCOR e hospitais auxiliares foram realizadas 9620 internações no ano de 1995, sendo que 903 (9,38\%) apresentavam IC. A maioria era do sexo masculino, 546(60,46\%) e os pacientes tinham idades que variaram de dois dias a 98 (média 52,68 $\pm 23,06$ ) anos.

Análise do diagnóstico principal identificou as seguintes cardiopatias (fig. 1): cardiopatia congênita 67 $(7,41 \%)$, HA $63(6,97 \%)$, miocardiopatia dilatada 233

\begin{tabular}{|c|c|c|c|}
\hline & Homens & Mulheres & \\
\hline $\mathrm{N}$ & $546(60,46 \%)$ & $357(39,50 \%)$ & $\mathrm{P}$ \\
\hline Idade (média e desvio padrão) & $54,21 \pm 20,41$ & $50,35 \pm 25,71$ & 0,0172 \\
\hline Óbito & $86(15,75 \%)$ & $62(14,57 \%)$ & 0,628 \\
\hline Cirurgia & $158(28,74 \%)$ & $132(36,97 \%)$ & 0,011 \\
\hline Card. congênita & $28(41,79 \%)$ & $39(58,21 \%)$ & \\
\hline Hip. arterial & $31(39,21 \%)$ & $32(60,79 \%)$ & \\
\hline Mioc. dilatada & $144(61,80 \%)$ & $89(38,20 \%)$ & \\
\hline Mioc. isquêmica & $217(73,56 \%)$ & $78(26,44 \%)$ & \\
\hline Mioc. restritiva & $7(46,67 \%)$ & $8(53,33 \%)$ & \\
\hline Valvopatia & $102(51,26 \%)$ & $97(48,74 \%)$ & 0,001 \\
\hline $\mathrm{AVC}$ & $16(2,93 \%)$ & $22(6,16 \%)$ & 0,018 \\
\hline BAVT & $25(4,58 \%)$ & $16(4,48 \%)$ & 0,945 \\
\hline Diabetes mellitus & $77(14,10 \%)$ & $37(10,36 \%)$ & 0,098 \\
\hline DPOC & $21(3,85 \%)$ & $3(0,84 \%)$ & 0,006 \\
\hline Fibrilação atrial & $60(10,99 \%)$ & $48(13,45 \%)$ & 0,266 \\
\hline Hipert. arterial & $60(18,99 \%)$ & $39(10,92 \%)$ & 0,976 \\
\hline Hip. pulmonar & $17(3,11 \%)$ & $16(4,48 \%)$ & 0,284 \\
\hline Inf. do miocárdio & $46(8,42 \%)$ & $18(6,04 \%)$ & 0,053 \\
\hline Ins. renal & $53(9,71 \%)$ & $29(8,12 \%)$ & 0,418 \\
\hline Inf. pulmonar & $51(9,34 \%)$ & $39(10,92 \%)$ & 0,437 \\
\hline Sem diagnóstico secundário & $227(41,59 \%)$ & $145(40,62 \%)$ & 0,775 \\
\hline
\end{tabular}




\begin{tabular}{|c|c|c|c|c|c|c|}
\hline IDADE EM ANOS & $<20$ & $20-39$ & $40-59$ & $60-79$ & $>80$ & $\mathrm{p}$ \\
\hline $\mathrm{N}$ & $121(13,29)$ & $94(10,40)$ & $280(31,00)$ & $354(39,20)$ & $54(5,98)$ & \\
\hline Mulheres & $69(59,02)$ & $33(35,11)$ & $95(33,93)$ & $135(38,14)$ & $25(46,30)$ & 0,001 \\
\hline Óbito & $34(28,10)$ & $17(18,09)$ & $29(10,36)$ & $47(13,28)$ & $11(20,37)$ & 0,001 \\
\hline Cirurgia & $41(33,88)$ & $40(42,55)$ & $90(32,14)$ & $110(31,07)$ & $9(16,67)$ & 0,027 \\
\hline Card. cong. & $45(67,16)$ & $9(13,43)$ & $10(14,93)$ & $2(2,99)$ & $1(1,49)$ & \\
\hline Hip. arterial & $1(1,59)$ & $7(11,11)$ & $18(28,57)$ & $29(46,03)$ & $8(12,70)$ & \\
\hline Mioc. dilatada & $27(11,59)$ & $31(13,30)$ & $87(37,34)$ & $69(29,26)$ & $19(8,15)$ & \\
\hline Mioc. isquêmica & $2(0,68)$ & $6(2,03)$ & $93(31,53)$ & $174(58,98)$ & $20(6,78)$ & \\
\hline Mioc. restritiva & $6(40,00)$ & $2(13,33)$ & $2(13,33)$ & $5(33,33)$ & 0 & \\
\hline Valvopatia & $32(16,08)$ & $35(17,59)$ & $66(33,17)$ & $65(32,66)$ & $1(0,50)$ & 0,001 \\
\hline $\mathrm{AVC}$ & $1(0,83)$ & $3(3,19)$ & $12(4,29)$ & $18(5,08)$ & $4(7,41)$ & 0,221 \\
\hline BAVT & $2(1,65)$ & $3(3,19)$ & $11(3,93)$ & $19(5,37)$ & $6(11,11)$ & 0,064 \\
\hline Diabetes mellitus & 0 & 0 & $31(11,07)$ & $71(20,06)$ & $12(22,22)$ & 0,001 \\
\hline DPOC & 0 & 0 & $5(1,79)$ & $18(5,09)$ & $1(1,85)$ & 0,005 \\
\hline Fibr. atrial & $4(3,31)$ & $6(6,39)$ & $40(14,29)$ & $50(14,12)$ & $8(14,81)$ & 0,005 \\
\hline Hip. arterial & 0 & $2(2,13)$ & $34(12,14)$ & $56(15,92)$ & $7(12,96)$ & 0,001 \\
\hline Hip. pulmonar & $10(8,26)$ & $7(7,45)$ & $10(3,57)$ & $5(1,41)$ & $1(1,35)$ & 0,002 \\
\hline Inf. do miocárdio & 0 & 0 & $20(7,14)$ & $41(11,58)$ & $3(5,56)$ & 0,001 \\
\hline Ins. renal & $7(5,79)$ & $4(4,26)$ & $25(8,93)$ & $40(11,30)$ & $6(11,1)$ & 0,157 \\
\hline Inf. pulmonar & $15(12,40)$ & $4(4,26)$ & $22(7,86)$ & $42(11,86)$ & $7(12,96)$ & 0,109 \\
\hline Sem diagnóstico secundário & $77(63,64)$ & $55(58,51)$ & $119(42,50)$ & $105(29,66)$ & $16(29,63)$ & 0,001 \\
\hline Com mais de 2 diagnósticos & $14(11,57)$ & $6(6,38)$ & $58(20,71)$ & $114(32,20)$ & $17(31,48)$ & \\
\hline
\end{tabular}

(25,8\%) doença de Chagas em 56 casos, miocardiopatia isquêmica 295 (32,66\%), infarto do miocárdio em 28 casos, miocardiopatia restritiva 15 (1,66\%), valvopatias 199 $(22,03 \%)$ e outras $31(3,43 \%)$.

Durante a internação, $290(32,11 \%)$ pacientes foram submetidos a procedimentos de intervenção sobre a sua cardiopatia de base, sendo 242 cirurgias propriamente ditas, 34 implantes de marcapasso, 10 angioplastias e quatro ablações de feixes anômalos (para tratamento da taquimiocardiopatia).

A distribuição dos casos segundo os diagnósticos secundários foi: acidente vascular cerebral, $38(4,20 \%)$; bloqueio atrioventricular (BAV) total, 41 (4,54\%); diabetes mellitus, 114 (12,62\%); doença pulmonar obstrutiva crônica, 24 (2,65\%); fibrilação atrial, 108 (11,96\%); HA, 99 (10,96\%); hipertensão pulmonar, 33 (33,65\%); infarto agudo do miocárdio, 64 (7,08\%); insuficiência renal, 82 (9,08\%); e infecção pulmonar, $90(9,96 \%)$. Em $531(58,81 \%)$ casos foram identificados diagnósticos secundários, nestes em 145 $(16,05 \%)$ dois diagnósticos, $52(5,75 \%)$ três diagnósticos e $12(1,32 \%)$ quatro ou mais diagnósticos. Ocorreram 138 $(15,28 \%)$ óbitos durante o período analisado.

A análise da influência do sexo (tab. I) demonstrou que os homens são em média mais velhos, mas as mulheres são mais freqüentes nos extremos de faixa etária $(\mathrm{p}=0,001)$.

A cardiopatia principal diferiu entre os sexos, com miocardiopatia dilatada $(61,80 \%$ vs $38,02 \%)$ e miocardiopatia isquêmica ( $73,56 \%$ vs $26,44 \%$ ), predominando entre os homens 144 e 217 casos, respectivamente, e cardiopatia congênita $(41,79 \%$ vs $58,21 \%)$ e miocardiopatia restritiva $(46,67 \%$ vs

\begin{tabular}{|c|c|c|c|c|c|c|c|}
\hline & $\mathrm{C}$ & $\mathrm{HA}$ & MD & MI & MR & $\mathrm{V}$ & Valor de $\mathrm{p}$ \\
\hline $\mathrm{n}$ & $67(7,41)$ & $63(6,97)$ & $233(25,80)$ & $295(32,66)$ & $15(1,66)$ & $199(22,03)$ & \\
\hline Idade média & $16,96+22,96$ & $62,68+16,10$ & $51,87+22,17$ & $64,16+12,12$ & $38,87+26,87$ & $47,20+20$ & 0,001 \\
\hline Mulheres & $39(58,21)$ & $32(50,79)$ & $89(38,20)$ & $78(26,44)$ & $8(53,33)$ & $97(48,74)$ & 0,001 \\
\hline Óbitos & $17(25,37)$ & $4(6,35)$ & $37(15,88)$ & $33(11,19)$ & $4(26,67)$ & $34(17,09)$ & 0,003 \\
\hline Cirurgia & $26(38,81)$ & $5(7,94)$ & $38(16,31)$ & $79(26,78)$ & $6(40,00)$ & $126(63,32)$ & 0,001 \\
\hline AVC & 0 & $6(9,52)$ & $7(3,00)$ & $15(5,08)$ & 0 & $5(4,52)$ & 0,145 \\
\hline BAVT & $1(1,49)$ & $3(4,76)$ & $28(12,02)$ & $4(1,36)$ & $05(2,51)$ & 0,001 & \\
\hline Diab.mellitus & $1(1,49)$ & $10(15,87)$ & $16(6,87)$ & $77(26,10)$ & 0 & $7(3,52)$ & 0,001 \\
\hline DPOC & 0 & $3(4,76)$ & $3(1,29)$ & $15(5,08)$ & 0 & $2(1,01)$ & 0,031 \\
\hline Fibr. atrial & $2(2,99)$ & $10(15,87)$ & $30(12,89)$ & $24(8,14)$ & $16(6,67)$ & $40(20,10)$ & 0,001 \\
\hline Hip. arterial & $3(4,48)$ & $63(100)$ & $6(2,58)$ & $73(24,75)$ & 0 & $13(6,53)$ & 0,001 \\
\hline Hip. pulmonar & $17(25,37)$ & $1(1,59)$ & $2(0,86)$ & $1(0,34)$ & 0 & $11(5,53)$ & 0,001 \\
\hline Ins. renal & $1(1,49)$ & $9(14,29)$ & $17(7,30)$ & $44(14,92)$ & 0 & $10(5,03)$ & 0,001 \\
\hline Inf. pulmonar & $8(11,94)$ & $6(9,52)$ & $35(15,02)$ & $24(8,14)$ & $1(6,67)$ & $10(5,03)$ & 0,011 \\
\hline Sem diagnóstico secundário & $34(50,75)$ & $24(38,10)$ & $107(45,92)$ & $75(25,42)$ & $11(73,33)$ & $102(51,26)$ & 0,001 \\
\hline
\end{tabular}

n- número de casos; AVC- acidente vascular cerebral; BAVT- bloqueio atrioventricular total; DPOC- doença pulmonar obstrutiva crônica; C- cardiopatia congênita; HA- hipertensão arterial; MD- miocardiopatia dilatada; MI- miocardiopatia isquêmica; MR- miocardiopatia restritiva; V- valvopatias. 
$53,33 \%$ ) entre as mulheres, 39 e 8 casos respectivamente. As mulheres foram mais freqüentemente submetidas à correção da cardiopatia de base $(36,97 \%$ vs $28,94 \%$; $p=0,011)$.

Conforme o sexo, houve diferença de incidência dentre os diagnósticos secundários: acidente vascular cerebral $(6,6 \%$ vs $2,3 \%$; $\mathrm{p}=0,01)$ mais freqüente nas mulheres e doença pulmonar obstrutiva crônica $(0,4 \%$ vs $3,85 \%$; $\mathrm{p}=0,006$ ), mais frequiente nos homens; os outros diagnósticos tiveram incidência similar (tab. I). A mortalidade foi semelhante em ambos os sexos.

Considerando a faixa etária (tab. II) predominaram na casuística pacientes entre 40 e 79 anos, correspondendo a $70,21 \%$ dos casos.

As cardiopatias congênitas foram mais freqüente nos mais jovens, a miocardiopatia isquêmica nos mais velhos, valvopatia e miocardiopatia dilatada no grupo intermediário.

Quanto aos diagnósticos secundários, o diabetes mellitus foi mais freqüente nas idades maiores; doença pulmonar obstrutiva crônica mais observada no grupo entre 60 e 79 anos; fibrilação atrial e HA observadas a partir dos 40 anos; hipertensão pulmonar mais freqüente nos <39 anos; infarto do miocárdio mais freqüente no grupo de 60 a 79 anos e infecção pulmonar nos dois extremos. Houve maior incidência de múltiplos diagnósticos com o aumento da idade, especialmente nos $>60$ anos e nos pacientes idosos foi menor o número de operados $(\mathrm{p}=0,022)$.

A mortalidade foi maior nos pacientes com idades $<20$ anos e $>80$ anos ( $\mathrm{p}=0,001)$.

Considerando a etiologia da cardiopatia (tab. III) verificou-se que a maioria das valvopatias teve tratamento cirúrgico, enquanto as outras cardiopatias foram menos ope$\operatorname{radas}(\mathrm{p}=0,001)$.

Houve maior frequiência de diabetes mellitus entre pacientes com miocardiopatia isquêmica, insuficiência renal entre hipertensos e portadores de miocardiopatia isquêmica; fibrilação atrial em portadores de valvopatias, BAV total entre portadores de miocardiopatia dilatada; infecção pulmonar entre pacientes com cardiopatia congênita ou miocardiopatia dilatada.

A mortalidade foi maior entre os portadores de cardiopatia congênita e miocardiopatia restritiva e menor naqueles $\operatorname{comHA}(\mathrm{p}=0,001)$.

\section{Discussão}

A IC vem se tornando um dos maiores problemas de saúde pública nos últimos anos, quer pelo aumento de sua incidência como pelos gastos que determina.

Segundo os dados do estudo Framingham ${ }^{3}$, a causa mais freqüente de IC é a HA. Dados de estudos multicêntricos, mais recentes identificam doença isquêmica como a etiologia mais freqüente, responsável por aproximadamente $70 \%$ dos $\operatorname{casos}^{7,8}$. No Brasil, suspeita-se que doença de Chagas seja uma causa freqüente, entretanto não temos dados sobre a real incidência de IC bem como de suas causas.

Como a IC é uma das maiores causas de internação hospitalar, procuramos estudar a freqüência e possíveis

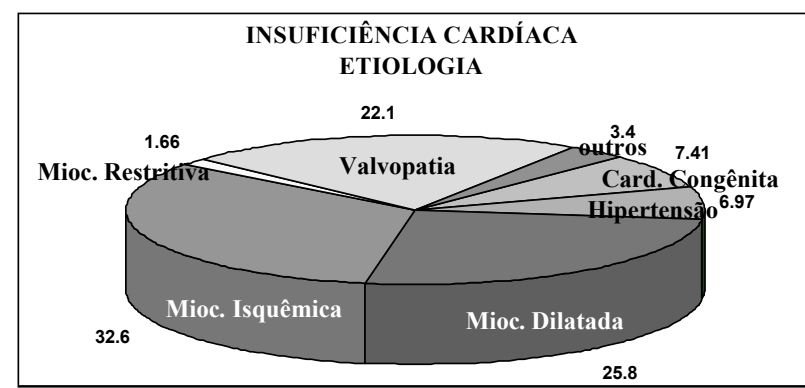

Fig. 1 - Gráfico mostrando as principais causas de insuficiência cardíaca dos pacientes internados no INCOR em 1995. Observar a maior incidência de miocardiopatia isquêmica.

causas de IC em hospital de referência de São Paulo, analisando os pacientes internados em 1995. Dentre o total das 9620 internações realizadas no INCOR, 903 apresentavam IC, ou seja 9,38\% dos internados apresentavam descompensação cardíaca de importância.

Esta freqüência parece diferir dos dados do $\mathrm{SUS}^{3}$, uma vez que mostra que em nossa Instituição, IC não é a maior causa de internação, pelo menos como diagnóstico principal. Sem dúvida a característica do hospital que privilegia terapêutica cirúrgica determina esta diferença.

De modo semelhante às casuísticas internacionais ${ }^{7,8}$, houve predominância de miocardiopatia isquêmica como causa da IC $(32,66 \%)$ nos casos que procuram por centro especializado (fig. 1).

A segunda causa foi a miocardiopatia dilatada com $25,3 \%$. A doença de Chagas foi causa da falência cardíaca em $6,2 \%$ da casuística global e de $24 \%$ no conjunto das miocardiopatias dilatadas, frequiência expressiva, especialmente considerando que São Paulo não é zona endêmica da doença. Este número pode ser ainda maior se considerarmos que somente os casos mais graves são internados, permitindo supor que doença de Chagas ainda é uma causa importante de IC e deve ser pesquisada.

A freqüência expressiva de valvopatias $(22,03 \%)$ e de cardiopatias congênitas $(7,41 \%)$ e baixa de HA $(6,95 \%)$ também se explicam pelas características da Instituição.

A etiologia da IC, como não poderia deixar de ser, varia conforme a faixa etária estudada, com predomínio absoluto de cardiopatia congênita nas baixas idades e de miocardiopatia isquêmica nos mais velhos. Valvopatia e miocardiopatia dilatada são mais freqüentes na faixa intermediária de 20 a 60 anos.

Dado interessante, que confirma os dados da literatura ${ }^{2,4}$, é a maior mortalidade observada nos extremos etários (>20\%). Esta tolerância reduzida da IC ocorre nas crianças pela gravidade da cardiopatia e nos idosos pela associação de múltiplas afecções (fig. 2).

De fato, doença associada foi observada em mais da metade dos pacientes, sem diferença entre sexos, mas com relação significativa com a idade (fig. 3). Entre os mais jovens 63,6\% não apresentaram diagnóstico secundário e nos >60 anos somente $29 \%$ não o apresentaram. Estes dados estão de acordo com os da literatura ${ }^{9}$, que mostram que pacientes idosos apresentam mais doenças associadas e em maior número. 


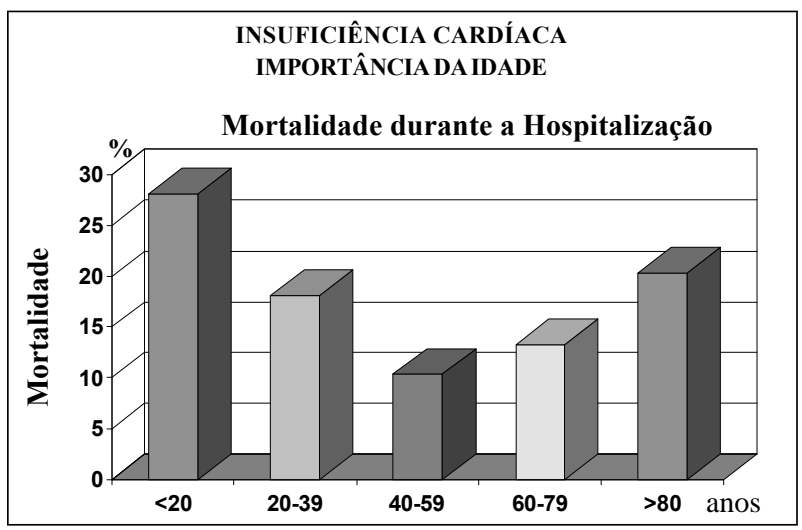

Fig. 2 - Gráfico de barras mostrando a maior mortalidade em crianças e idosos.

Consideradas as doenças associadas mais freqüentes, infecção pulmonar predominou nas crianças, provavelmente influindo para maior mortalidade nessa faixa etária.

Por outro lado, fibrilação atrial, insuficiência renal e infecção pulmonar foram mais freqüentes com o aumento da idade e diabetes mellitus mais freqüente nos mais velhos. Estas condições reconhecidamente agravam a IC e, provavelmente, contribuíram para a necessidade de internação e maior mortalidade nas faixas etárias maiores.

Análise dos dados segundo a cardiopatia, mostra-nos que diabetes foi mais freqüente entre os pacientes com miocardiopatia isquêmica e com HA, conforme o esperado, pela sua relação epidemiológica e etiopatogênica ${ }^{10}$. Fibrilação atrial foi observada com maior frequiência nos portadores de valvopatias, também esperado, devido ao elevado número de casos com valvopatia mitral (96 casos ou 48,24\%). Insuficiência renal foi mais freqüente nos pacientes com HA e coronariopatia, provavelmente pela arteriopatia comum a estas doenças.

$\mathrm{O}$ achado de mortalidade maior nos portadores de cardiopatia congênita e de miocardiopatias restritivas pode ser explicado nos primeiros pela complexidade e nos últimos pelo pior prognóstico inerente a endomiocardiofibrose, amiloidose e miocardiopatia restritiva.

Considerando-se o número de casos operados, observou-se que o seu percentual foi relativamente pequeno, diferindo conforme a cardiopatia. Os dados ressaltam a importância do diagnóstico das valvopatias, pois na maioria dos casos, foi possível a correção cirúrgica.

Dado interessante observado foi o maior percentual de mulheres submetido a cirurgias, pois na literatura ${ }^{11}$, as mu-

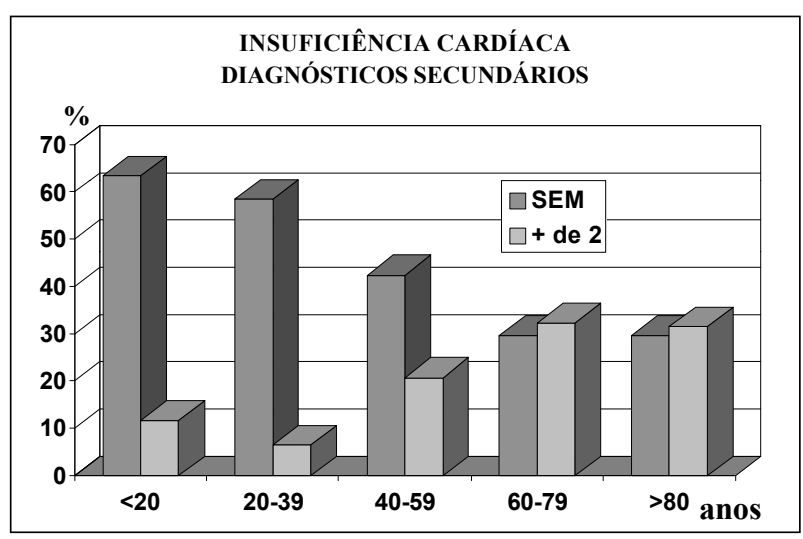

Fig. 3 - Gráfico de barras mostrando o aumento de diagnósticos secundários com o aumento da idade.

lheres são menos estudadas e menos submetidas às correções cirúrgicas.

Entre os pacientes com coronariopatia, contudo, o percentual de operados foi a metade dos observados entre os valvopatas (38,81\% vs $63,32 \%)$. Provavelmente, as condições cardiológicas e gerais dos pacientes não permitiram a realização de cirurgia. $\mathrm{O}$ mesmo se aplica às cardiopatias congênitas que, devido a sua complexidade em muitos casos, não permite esta correção.

Enfim, podemos concluir que em pacientes internados num hospital terciário eminentemente dirigido para tratamento cirúrgico, IC foi descrita em $9,38 \%$ dos pacientes e a análise desta população permitindo-nos as seguintes observações: a causa mais freqüente foi miocardiopatia isquêmica, seguida por miocardiopatia dilatada; em 32,11\% foi possível corrigir a causa da IC, especialmente nos valvopatas; diagnósticos secundários ou fatores agravantes foram observados em mais da metade dos casos, diferindo conforme a cardiopatia de base, e em maior número nos > 60 anos; a mortalidade foi maior nas crianças, devendo-se, provavelmente, a maior complexidade da cardiopatia e nos >80 anos, à maior associação de diagnósticos secundários ou fatores agravantes.

O conhecimento destes dados auxilia-nos na condução dos casos de IC, possibilitando um melhor tratamento e maior atenção, especialmente, nos grupos de maior risco e, quem sabe, possibilitando interferir na evolução da IC, e reduzindo a sua morbidade e mortalidade, quer através de tratamento clínico ou cirúrgico, sempre considerando a importância do diagnóstico precoce e do controle das doenças associadas.

\section{Referências}

1. Parameshawar J, Shackell MM, Richardson A et al - Prevalence of heart failure in three general practices in north west London. J Gen pract 1992; 42: 287-9.

2. Schocken DD, Arrieta MI, Leaverton PE et al - Prevalence and mortality rate of congestive heart failure in the United States. J Am Coll Cardiol 1992; 20: 301-6.

3. Ministério da Saúde. Coordenação de Doenças Cardiovasculares no Brasil. Sistema Único de Saúde - SUS Brasília 1993, pag 32.
4. Ho KKL, Pinsky JL, Kannel WB et al - The epidemiology of heart failure: The Framingham Study. J Am Coll Cardiol 1993; 22 (supp A): 6A-13A.

5. McMurray J, Dargie HJ - Trends in hospitalisation for chronic heart failure in the United Kingdom. Eur Heart J 1992; 13(supp): 350.

6. Sutton G - Epidemiologic aspects of heart failure. Am Heart J 1990; 120: 1538-40.

7. The SOLVD Investigators - Effect of enalapril on survival in patients with 
reduced left ventricular ejection fractions and congestive heart failure. N Engl J Med 1991; 325: 293-302.

8. The Digitalis Investigation Group - Rationale, design, implementation and baseline characteristics of patients in the DIG trial: a large, simple long-term trial to evaluate the effect of digitalis on mortality in heart failure. Control Clin Trials 1996; 17: 77-97.

9. Guccione AA, Felson DT, Anderson JJ te al - The effects of specific medical conditions on the functional limitations of elders in the Framingham Study. Am J Public Health 1994; 84: 351-8.

10. Farmer JA, Gotto AM - Dyslipidemia and other risk factors for coronary artery disease, in Braunwald E (ed); Heart Disease. A textbook of cardiovascular Medicine. Philadelphia, W B Saunders Company, 1997, pp 1126-60.

11. Ayanian JZ, Epstein AM - Differences in the use of procedures between women and men hospitalized for coronary disease. N Engl J Med 1991; 325: 221-6. 